\title{
NONEXISTENCE OF GENERALIZED SCATTERING RAYS AND SINGULARITIES OF THE SCATTERING KERNEL FOR GENERIC DOMAINS IN $\mathbb{R}^{3}$
}

\author{
LUCHEZAR STOJANOV
}

(Communicated by Frederick R. Cohen)

\begin{abstract}
It is proved for fixed unit vectors $\omega \neq \theta$ in $\mathbb{R}^{3}$ and generic bounded open domains $\mathfrak{D} \subset \mathbb{R}^{3}$ that there do not exist generalized $(\omega, \theta)$ rays in $\Omega=\mathbb{R}^{3} \backslash \mathfrak{D}$ containing nontrivial geodesics on $\partial \Omega$. Consequently, for generic domains the sojourn times of reflecting $(\omega, \theta)$-rays completely describe the set of singularities of the scattering kernel $s(t, \theta, \omega)$.
\end{abstract}

\section{INTRODUCTION}

Let $\Omega$ be a closed domain in $\mathbb{R}^{3}$ with $C^{\infty}$ smooth boundary $\partial \Omega$ and bounded complement $\mathfrak{D}=\mathbb{R}^{3} \backslash \Omega$. For fixed $\omega, \theta \in S^{2}$ the scattering kernel $s(t, \theta, \omega)=s_{\Omega}(t, \theta, \omega)$ related to the wave equation in $\mathbb{R} \times \Omega$ with Dirichlet boundary conditions on $\mathbb{R} \times \partial \Omega$ is a distribution in $\mathscr{S}^{\prime}\left(\mathbb{R}_{t}\right)$ (see [6] for the definition). It was suggested by Guillemin [3] that the analysis of the singularities of $s(t, \theta, \omega)$ is connected with the sojourn times of the $(\omega, \theta)$-rays in $\Omega$.

Let $\gamma: \mathbb{R} \rightarrow \Omega$ be a generalized geodesic in $\Omega$; i.e. $\gamma=\imath \circ \tilde{\gamma}$, where $\tilde{\gamma}: \mathbb{R} \rightarrow$ $T^{*}(\mathbb{R} \times \Omega)$ is a generalized bicharacteristic of the wave operator $\square=\partial_{t}^{2}-\Delta$ (see [7] or [4, §24.3]) and $l: T^{*}(\mathbb{R} \times \Omega) \rightarrow \Omega$ is the canonical projection. If there exist real numbers $a<b$ such that $\dot{\gamma}(t)=\omega$ for $t \leq a$ and $\dot{\gamma}(t)=\theta$ for $t \geq b$, then $\gamma$ (and sometimes $\operatorname{Im} \gamma$ ) is called a $(\omega, \theta)$-ray in $\Omega$. Such a curve $\gamma$ consists of linear segments in $\Omega$ (two of them are infinite straightline rays) and gliding segments (i.e. geodesics with respect to the standard metric) on $\partial \Omega$. If $\operatorname{Im} \gamma$ contains only a finite number of linear segments and does not contain gliding ones, then $\gamma$ is called a reflecting $(\omega, \theta)$-ray in $\Omega$, otherwise $\gamma$ is called a generlized $(\omega, \theta)$-ray. By $\mathfrak{L}_{\omega, \theta}=\mathfrak{L}_{\omega, \theta}(\Omega)$ we denote the set of all $(\omega, \theta)$-rays in $\Omega$.

Received by the editors January 8, 1990 and, in revised form, May 8, 1990.

1980 Mathematics Subject Classification (1985 Revision). Primary 58G25, 58F30; Secondary $58 \mathrm{~F} 17$.

Key words and phrases. Generalized and reflecting $(\omega, \theta)$-rays, sojourn time, scattering kernel, generic.

This work was partially supported by the Bulgarian Committee of Sciences, Grant 52 . 
Fix an open ball $B$ with radii $a>0$ that contains $\mathfrak{D}$. For $\eta \in S^{2}$ let $Z_{\eta}$ be the hyperplane in $\mathbb{R}^{3}$ tangent to $B$ such that $Z_{\eta}$ is orthogonal to $\eta$ and the halfspace $H_{\eta}$, determined by $Z_{\eta}$ and having $\eta$ as an inward normal, contains $B$. For a $(\omega, \theta)$-ray $\gamma$ in $\Omega$ denote by $T_{\gamma}^{\prime}$ the length of this part of $\gamma$ that is contained in $H_{\omega} \cap H_{-\theta}$. Then $T_{\gamma}=T_{\gamma}^{\prime}-2 a$ is called the sojourn time of $\gamma$ (cf. Guillemin [3]). It is easy to see that the definition of $T_{\gamma}$ does not depend on the choice of the ball $B$.

Under some assumptions on $\Omega$ it was established in [10] that

$$
\operatorname{sing} \operatorname{supp} s(t, \theta, \omega) \subset\left\{-T_{\gamma}: \gamma \in \mathfrak{L}_{\omega, \theta}\right\},
$$

where $\mathfrak{L}_{\omega, \theta}=\mathfrak{L}_{\omega, \theta}(\Omega)$ is the set of all $(\omega, \theta)$-rays in $\Omega$. Moreover, in [10] a formula was proved for the main singularity of $s(t, \theta, \omega)$ for $t$ close to some $T \in \operatorname{sing} \operatorname{supp} s(t, \theta, \omega)$. Recently, the inclusion (1) was established in [1] under weaker assumptions, and it was also shown there that for generic $\Omega$ in $\mathbb{R}^{n}, T_{\gamma} \in \operatorname{sing} \operatorname{supp} s(t, \theta, \omega)$ for a class of reflecting $(\omega, \theta)$-rays $\gamma$ in $\Omega$. In $[8,9,14]$ all singularities of $s(t, \theta, \omega)$ have been examined for special classes of obstacles $\mathfrak{D}$.

For $X=\partial \Omega$ denote by $C^{\infty}\left(X, \mathbb{R}^{3}\right)$ the space of all $C^{\infty}$ maps of $X$ into $\mathbb{R}^{3}$ endowed with the Withney $C^{\infty}$ topology (cf. [2]), and by $\mathbf{C}(X)=C_{\mathrm{emb}}^{\infty}\left(X, \mathbb{R}^{3}\right)$ its open subset consisting of all $C^{\infty}$ embeddings of $X$ into $\mathbb{R}^{3}$. Then $\mathbf{C}(X)$ is a Baire topological space, so every residual subset (i.e. a countable intersection of open dense subsets), is dense in it. Given $f \in \mathbf{C}(X)$ we denote by $\Omega_{f}$ the unbounded closed domain in $\mathbb{R}^{3}$ with $\partial \Omega_{f}=f(X)$.

The main result in this paper is the following:

Theorem 1.1. Let $\theta \neq \omega$ be fixed unit vectors in $\mathbb{R}^{3}$. Then there exists a residual subset $\mathscr{R}$ of $\mathbf{C}(X)$ such that for each $f \in \mathscr{R}$ there are no generalized $(\omega, \theta)$ rays in $\Omega_{f}$, and

$$
\operatorname{sing} \operatorname{supp} s_{\Omega_{f}}(t, \theta, \omega)=\left\{-T_{\gamma}: \gamma \in \mathfrak{L}_{\omega, \theta}\left(\Omega_{f}\right)\right\} .
$$

Moreover, for $f \in \mathscr{R}$ and $\gamma \in \mathfrak{L}_{\omega, \theta}\left(\Omega_{f}\right)$ the main singularity of $s_{\Omega_{f}}(t, \theta, \omega)$ for $t$ near $-T_{\gamma}$ is given by the same formula as that in $[10,1]$ (see [1, Theorem 2]).

\section{Degenerate $(\omega, \theta)$-RAYS}

Let $\Omega$ be as in the introduction, $X=\partial \Omega$, and $\omega \in S^{2}$.

A curve $\gamma$ in $\Omega$ is called a degenerate $\omega$-ray if it has the form $\gamma=\bigcup_{i=0}^{k-1} l_{i} \subset$ $\Omega$ and the following conditions are satisfied:

(i) $l_{0}$ is the infinite linear segment starting at $x_{1}$ having direction $-\omega$;

(ii) for every $i=1, \ldots, k-1, l_{i}$ is a linear segment $\left[x_{i}, x_{i+1}\right], x_{i} \in X$ for $i=1, \ldots, k$;

(iii) if $k \geq 2$ then for any $i=1, \ldots, k-2$, the segments $l_{i}$ and $l_{i+1}$ satisfy the reflection law at $x_{i+1}$ with respect to $X$; i.e., $l_{i}$ and $l_{i+1}$ make equal acute 
angles with the interior (with respect to $\Omega$ ) unit normal vector $\nu\left(x_{i+1}\right)$ to $X$ at $x_{i+1}$ and $l_{i}, l_{i+1}, \nu\left(x_{i+1}\right)$ lie in a common plane; and

(iv) $l_{k-1}$ is tangent to $X$ at $x_{k}$, determining an asymptotic direction for $X$ at $x_{k}$ (cf. [15] for the definition of asymptotic direction).

The points $x_{1}, \ldots, x_{k}$ are called vertices of $\gamma$. If every segment of $\gamma$ is not tangent to $X$, then $\gamma$ is called ordinary. The defect of such a ray $\gamma$ is defined by $d(\gamma)=k-s$, where $s=\operatorname{card}\left\{x_{1}, \ldots, x_{k}\right\}$.

Note that if the curvature of $X$ does not vanish of infinite order, then for every generalized $(\omega, \theta)$-ray $\gamma$ in $\Omega$ there exist a degenerate $\omega$-ray $\gamma_{1}$ and a degenerate $(-\theta)$-ray $\gamma_{2}$ with $\gamma_{i} \subset \operatorname{Im} \gamma, i=1,2$ (cf. [7]).

For a set $A$ and an integer $s \geq 2$ we use the notation:

$$
A^{(s)}=\left\{\left(a_{1}, \ldots, a_{s}\right) \in A^{s}: a_{i} \neq a_{j} \text { for } i \neq j\right\} .
$$

Lemma 2.1. There exists a residual subset $\mathfrak{R}(\omega)$ of $\mathbf{C}(X)$ such that for $f \in \mathfrak{R}(\omega)$ if $\gamma$ is a degenerate $\omega$-ray in $\Omega_{f}$, then $d(\gamma)=0$.

To prove the assertion one can use some arguments from the proof of Lemma 2.2 as well as a combinatorial classification of the degenerate $\omega$-rays, similar to that for periodic reflecting rays used in $\S 4$ of [11]. Since the modifications are rather standard, we omit the details.

For an integer $k \geq 1$ and $\omega \in S^{2}$, denote by $\mathscr{D}(\omega ; k)$ the set of those $f \in$ $\mathbf{C}(X)$ such that the set of all $y=\left(y_{1}, \ldots, y_{k}\right) \in f(X)^{(k)}$ for which $y_{1}, \ldots, y_{k}$ are the successive vertices of a degenerate $\omega$-ray on $f(X)$ is a discrete subset of $f(X)^{(k)}$.

Lemma 2.2. The set $\mathscr{D}(\omega ; k)$ contains a residual subset of $\mathbf{C}(X)$.

Proof. To prove the assertion it is sufficient to establish that $\mathscr{D}(\omega ; k) \cap$ $C_{\mathrm{emb}}^{\infty}\left(X, H_{\omega}\right)$ contains a residual subset of $C_{\mathrm{emb}}^{\infty}\left(X, H_{\omega}\right)$.

We proceed as in [11-13]. Let $\pi: \mathbb{R}^{3} \rightarrow Z=Z_{\omega}$ be the orthogonal projection. Denote by $U_{k}$ the set of those $y=\left(y_{1}, \ldots, y_{k}\right) \in\left(\mathbb{R}^{3}\right)^{(k)}$ such that for every $i=1, \ldots, k-1, y_{i}$ does not belong to the segment $\left[y_{i-1}, y_{i+1}\right]$, where by definition $y_{0}=\pi\left(y_{1}\right)$. Define $F: U_{k} \rightarrow \mathbb{R}$ by

$$
F(y)=\sum_{i=0}^{k-1}\left\|y_{i}-y_{i+1}\right\| .
$$

If $y_{1}, \ldots, y_{k}$ are the successive reflection points of a degenerate $\omega$-ray $\gamma$ in $\Omega_{f}$ with $d(\gamma)=0$, then $y=\left(y_{1}, \ldots, y_{k}\right) \in U_{k}$ and $F(y)$ is the length of $\gamma \cap H_{\omega}$. Moreover, for $y^{\prime}=\left(y_{1}, \ldots, y_{k-1}\right)$ we have $\operatorname{grad}_{y^{\prime}} F(y)=0$, $\left\langle y_{k}-y_{k-1}, \nu\left(y_{k}\right)\right\rangle=0$, and $w=y_{k}-y_{k-1}$ is an asymptotic direction for $Y$ at $y_{k}$. The last condition can be expressed analytically as follows: Let $r: V \rightarrow Y$ be a chart, where $V$ is an open subset of $\mathbb{R}^{2}$ and $r(V)$ is an open neighborhood of $y_{k}$ in $Y$. Then $w=\lambda\left(\partial r / \partial u_{1}\right)(u)+\mu\left(\partial r / \partial u_{2}\right)(u)$ for some $\lambda, \mu \in \mathbb{R}$, where $r(u)=y_{k}, u=\left(u_{1}, u_{2}\right)$. Let $L, M, N$ be the coefficients of the 
second fundamental form of $Y$ at $y_{k}$; that is, $L(u)=\left\langle\left(\partial^{2} r / \partial u_{1}^{2}\right)(u), \nu\left(y_{k}\right)\right\rangle$, $M(u)=\left\langle\left(\partial^{2} r / \partial u_{1} \partial u_{2}\right)(u), \nu\left(y_{k}\right)\right\rangle, N(u)=\left\langle\left(\partial^{2} r / \partial u_{2}^{2}\right)(u), \nu\left(y_{k}\right)\right\rangle$. Then $w$ is an asymptotic direction for $Y$ at $y_{k}$ iff (cf. [15])

$$
L \lambda^{2}+2 M \lambda \mu+N \mu^{2}=0 \text {. }
$$

It is easy to check that $\lambda=\left\langle w,\left(G \partial r / \partial u_{1}-F \partial r / \partial u_{2}\right) /\left(E G-F^{2}\right)\right\rangle$ and $\mu=$ $\left\langle w,\left(E \partial r / \partial u_{2}-F \partial r / \partial u_{1}\right) /\left(E G-F^{2}\right)\right\rangle$, where $E(u)=\left\|\left(\partial r / \partial u_{1}\right)(u)\right\|^{2}, F(u)=$ $\left\langle\left(\partial r / \partial u_{1}\right)(u),\left(\partial r / \partial u_{2}(u)\right\rangle\right.$, and $G(u)=\left\|\left(\partial r / \partial u_{2}\right)(u)\right\|^{2}$ are the coefficients of the first fundamental form. Therefore $(3)$ is equivalent to

$$
\begin{aligned}
& L\left\langle w, G \partial r / \partial u_{1}-F \partial r / \partial u_{2}\right\rangle^{2} \\
& \quad+2 M\left\langle w, G \partial r / \partial u_{1}-F \partial r / \partial u_{2}\right\rangle\left\langle w, E \partial r / \partial u_{2}-F \partial r / \partial u_{1}\right\rangle \\
& \quad+N\left\langle w, E \partial r / \partial u_{2}-F \partial r / \partial u_{1}\right\rangle^{2}=0 .
\end{aligned}
$$

Let $J_{k}^{2}\left(X, \mathbb{R}^{3}\right)$ be the $k$-fold bundle of 2-jets (cf. [2]). Given $f \in C^{\infty}\left(X, \mathbb{R}^{3}\right)$, the map $j_{k}^{2} f: X^{(k)} \rightarrow J_{k}^{2}\left(X, \mathbb{R}^{3}\right)$ is defined by $j_{k}^{2} f\left(x_{1}, \ldots, x_{k}\right)=\left(j^{2} f\left(x_{1}\right), \ldots\right.$, $\left.j^{2} f\left(x_{k}\right)\right)$. Here $j^{2} f(x) \in J^{2}\left(X, \mathbb{R}^{3}\right)$ is the 2-jet determined by $f$ at $x \in X$. Denote by $M$ the set of those $\tau=\left(j^{2} f_{1}\left(x_{1}\right), \ldots, j^{2} f_{k}\left(x_{k}\right)\right) \in J_{k}^{2}\left(X, \mathbb{R}^{3}\right)$ such that $\left(x_{1}, \ldots, x_{k}\right) \in X^{(k)} ;\left(f_{1}\left(x_{1}\right), \ldots, f_{k}\left(x_{k}\right)\right) \in U_{k} ; \operatorname{rank} d f_{i}\left(x_{i}\right)=2$ for every $i=1, \ldots, k$; and $f_{i}\left(x_{i}\right)-f_{i+1}\left(x_{i+1}\right)$ is not tangent to $f_{i}(X)$ at $f_{i}\left(x_{i}\right)$ for all $i=1, \ldots, k-1$, and $\omega$ is not tangent to $f_{1}(X)$ at $f_{1}\left(x_{1}\right)$. Then $M$ is open in $J_{k}^{2}\left(X, \mathbb{R}^{3}\right)$. Finally, define the singularity set $\Sigma$ as the set of those $\tau \in M$ such that $\operatorname{grad}_{x^{\prime}} F \circ\left(f_{1} \times \cdots \times f_{k}\right)(x)=0,\left\langle f_{k}\left(x_{k}\right)-f_{k-1}\left(x_{k-1}\right), \nu\right\rangle=0$, and $f_{k}\left(x_{k}\right)-f_{k-1}\left(x_{k-1}\right)$ is an asymptotic direction for $f_{k}(X)$ at $f_{k}\left(x_{k}\right)$, where $\nu$ is a nonzero normal vector to $f_{k}(X)$ at $f_{k}\left(x_{k}\right)$.

Next, using some arguments from [11] or [12] (cf., for example, [11, proof of Lemma 7.1]) we establish that $\Sigma$ is a smooth submanifold of $M$ with $\operatorname{codim} \Sigma=2 k$. Then for $f \in C^{\infty}\left(X, \mathbb{R}^{3}\right), j_{k}^{2} f \pitchfork \Sigma$ implies that $\{x \in$ $\left.X^{(k)}: j_{k}^{2} f(x) \in \Sigma\right\}$ is a discrete subset of $X^{(k)}$; i.e., $f \in \mathscr{D}(\omega ; k)$. Consequently $\mathscr{D}(\omega ; k)$ contains the residual subset:

$$
\left\{f \in C^{\infty}\left(X, \mathbb{R}^{3}\right): j_{k}^{2} f \pitchfork \Sigma\right\} \cap C_{\text {emb }}^{\infty}\left(X, H_{\omega}\right) \cap \Re(\omega)
$$

of $C_{\mathrm{emb}}^{\infty}\left(X, H_{\omega}\right)$. This proves the assertion.

\section{Nonexistence of GeNeralized $(\omega, \theta)$-RAys}

In this section we prove that generic domains $\Omega$ do not admit generalized $(\omega, \theta)$-rays. To this end we combine Lemma 2.2 with a simple perturbation technique.

Lemma 3.1. Let $X$ be a smooth surface in $\mathbb{R}^{3}$ and $c:[a, b] \rightarrow X$ be a geodesic on $X(b>a)$. Let $c\left(t_{0}\right)$ be an arbitrary point on the geodesic $\left(a<t_{0}<b\right)$ that is not a point of selfintersection, and $U$ be an arbitrary neighborhood of $c\left(t_{0}\right)$ 
in $X$ such that

$$
U \cap \operatorname{Im} c=\{c(t): \alpha<t<\beta\}
$$

for some $\alpha, \beta \in(a, b)$. Then there exists $f \in \mathbf{C}(X)$ arbitrarily close to id with respect to the $C^{\infty}$ topology such that supp $f \subset U$, and if $\tilde{c}:[a, b] \rightarrow \tilde{X}$ is the geodesic on $\tilde{X}=f(X)$ with $\tilde{c}(t)=c(t)$ for $t \in[a, \alpha]$, then

$$
\{\tilde{c}(t): t \in(\alpha, \beta]\} \cap\{c(t): t \in(\alpha, \beta]\}=\varnothing .
$$

Proof. We may assume that $U$ is small enough so that there exists coordinates $x_{0}, x_{1}$ in $U$ given by a chart $r: V \rightarrow U \subset X$, where $V=(\alpha, \beta) \times(-\delta, \delta) \subset \mathbb{R}^{2}$ for some $\delta>0, a<\alpha<t_{0}<\beta<b$, such that the components $g_{i j}$ of the standard metric $g$ on $X$ have the form:

$$
g_{00}\left(x_{0}, x_{1}\right)=1, \quad g_{01}\left(x_{0}, x_{1}\right)=0, \quad g_{11}\left(x_{0}, x_{1}\right)=G\left(x_{0}, x_{1}\right)>0
$$

for $\left(x_{0}, x_{1}\right) \in V$. Moreover, we may assume

$$
G\left(x_{0}, x_{1}\right)<1 \text { for all }\left(x_{0}, x_{1}\right) \in V \text {. }
$$

Otherwise we can replace $r$ by another chart, $\tilde{r}: V \rightarrow X$ given by $\tilde{r}\left(x_{0}, x_{1}\right)=$ $r\left(x_{0}, \varepsilon x_{1}\right)$; then $\tilde{g}_{11}\left(x_{0}, x_{1}\right)=\varepsilon^{2} g_{11}\left(x_{0}, x_{1}\right)<1$ for $\varepsilon>0$ sufficiently small. Moreover, (4) holds provided $t_{0}-\alpha, \beta-t_{0}$, and $\delta$ are sufficiently small. Also note that $r(t, 0)=c(t)$ for $t \in(\alpha, \beta)$.

Take arbitrary $C^{\infty}$ functions $\lambda, \mu: \mathbb{R} \rightarrow[0,1]$ with

$$
\operatorname{supp} \lambda=[\alpha, \beta], \quad p=\mu(0)>0, \quad q=\mu^{\prime}(0)>0 .
$$

For $\varepsilon>0$ small enough set $f_{\varepsilon}(y)=y$ for $y \in X \backslash U$ and $f_{\varepsilon}(y)=r(x)+$ $\varepsilon \lambda\left(x_{0}\right) \mu\left(x_{1}\right)\left(\partial r / \partial x_{0}\right)(x)$ for $y=r(x), x=\left(x_{0}, x_{1}\right) \in V$. Let $X_{\varepsilon}=f_{\varepsilon}(X)$. Then $\psi(x)=r(x)+\varepsilon \lambda\left(x_{0}\right) \mu\left(x_{1}\right)\left(\partial r / \partial x_{0}\right)(x)$ defines a chart, $\psi: V \rightarrow \psi(V) \subset$ $X_{\varepsilon}$. Let $g(\varepsilon)$ be the standard metric on $X_{\varepsilon}$ induced by $\mathbb{R}^{3}$; then for its components $g_{i j}\left(\varepsilon ; x_{0}, x_{1}\right)$ we have:

$$
\begin{aligned}
g_{00}\left(\varepsilon ; x_{0}, x_{1}\right)= & 1+2 \varepsilon \lambda^{\prime}\left(x_{0}\right) \mu\left(x_{1}\right)+O\left(\varepsilon^{2}\right), \\
g_{01}\left(\varepsilon ; x_{0}, x_{1}\right)= & \varepsilon \lambda\left(x_{0}\right) \mu^{\prime}\left(x_{1}\right)+O\left(\varepsilon^{2}\right), \\
g_{11}\left(\varepsilon ; x_{0}, x_{1}\right)= & G\left(x_{0}, x_{1}\right)+2 \varepsilon \lambda\left(x_{0}\right) \mu\left(x_{1}\right) \\
& \cdot\left\langle\left(\partial r / \partial x_{1}\right)(x),\left(\partial^{2} r / \partial x_{0} \partial x_{1}\right)(x)\right\rangle+O\left(\varepsilon^{2}\right)
\end{aligned}
$$

for $\varepsilon$ close to 0 .

Using $\left(x_{0}, x_{1}\right)$ consider the canonical coordinates $x_{0}, x_{1}, y_{0}, y_{1}$ in $T^{*} X_{\varepsilon}$ and the Hamiltonian vectorfield generated by the Hamiltonian:

$$
H(\varepsilon, x, y)=g_{00}(\varepsilon ; x) y_{0}^{2} / 2+g_{01}(\varepsilon ; x) y_{0} y_{1}+g_{11}(\varepsilon ; x) y_{1}^{2} / 2 \text {, }
$$

where $x=\left(x_{0}, x_{1}\right), y=\left(y_{0}, y_{1}\right)$. Let $c(\varepsilon ; t), 0 \leq t$, be the geodesic on $X_{\varepsilon}$ with $c(\varepsilon ; t)=c(t)$ for each $t \in[0, \alpha]$, and $\left(x^{(\varepsilon)}(t), y^{(\varepsilon)}(t)\right)$ be the corresponding integral curve in $T^{*} X_{\varepsilon}$. Writing the Hamiltonian equations for this curve, and then the corresponding variational equations for

$$
X_{i}(t)=\left.(d / d \varepsilon) x_{i}^{(\varepsilon)}(t)\right|_{\varepsilon=0}, \quad Y_{i}(t)=\left.(d / d \varepsilon) y_{i}^{(\varepsilon)}(t)\right|_{\varepsilon=0},
$$


we get (cf. (7)):

$$
\begin{aligned}
\dot{X}_{0}(t) & =Y_{0}(t)+2 p \lambda^{\prime}(t) \\
\dot{X}_{1}(t) & =G(t, 0) Y_{1}(t)+q \lambda(t) \\
\dot{Y}_{0}(t) & =-p \lambda^{\prime \prime}(t) \\
\dot{Y}_{1}(t) & =-q \lambda^{\prime}(t) \\
X_{0}(\alpha) & =X_{1}(\alpha)=Y_{0}(\alpha)=Y_{1}(\alpha)=0
\end{aligned}
$$

for $(\alpha \leq t \leq \beta)$. Consequently, $Y_{1}(t)=-q \lambda(t)$ and $\dot{X}_{1}(t)=q \lambda(t)(1-G(t, 0))$. Hence (6) yields $\dot{X}_{1}(t)>0$ for every $t \in(\alpha, \beta)$, and therefore, $X_{1}(t)>0$ for each $t \in(\alpha, \beta]$. This means that $(d / d \varepsilon) x_{1}^{(\varepsilon)}(t)>0$ for $t \in(\alpha, \beta]$, provided $\varepsilon>0$ is sufficiently small. Fix such an $\varepsilon$. Then $x_{1}^{(\varepsilon)}(t)$ is positive for $t \in(\alpha, \beta]$, and for $f=f_{\varepsilon}, \widetilde{X}=X_{\varepsilon}$, and $\tilde{c}(t)=c(\varepsilon, t)$ we have (5). This proves the assertion.

Fix two unit vectors $\omega \neq \theta$ in $\mathbb{R}^{3}$.

Theorem 3.2. There exists a residual subset $\mathscr{V}$ of $\mathbf{C}(X)$ such that for every $f \in \mathscr{V}$ there are no generalized $(\omega, \theta)$-rays in $\Omega_{f}$.

Proof. We are going to construct by induction a decreasing sequence $\mathscr{V}_{1} \supset \mathscr{V}_{2} \supset$ $\cdots \supset \mathscr{V}_{k} \supset \cdots$ of residual subsets of $\mathbf{C}(X)$ such that for any $k$ and any $f \in \mathscr{V}_{k}$ there are no generalized $(\omega, \theta)$-rays in $\Omega_{f}$ with not more than $k$ vertices.

It follows by [5] that there exists a residual subset $\mathscr{K}$ of $\mathbf{C}(X)$ such that whenever $f \in \mathscr{K}$, for every $y \in f(X)$ the curvature of $f(X)$ at $y$ does not vanish of third order with respect to any direction tangent to $f(X)$ at $y$. Then for $f \in \mathscr{K}$, if $\gamma: \mathbb{R} \rightarrow \Omega_{f}$ is a $(\omega, \theta)$-ray, then $\operatorname{Im} \gamma=\bigcup_{i=0}^{k} l_{i}$, where $l_{0}$ and $l_{k}$ are infinite segments, starting at $x_{1}$ and $x_{k}$, respectively, with directions $-\omega$ and $\theta$, and for any $i=1, \ldots, k-1, l_{i}$ is either a linear segment $\left[x_{i}, x_{i+1}\right]$ in $\Omega_{f}$ or a geodesic $x_{i} x_{i+1}$ on $\partial \Omega_{f}=f(X)$, and $x_{i}=\partial \Omega_{f}$ for every $i=1, \ldots, k$. Moreover, two successive linear segments of $\gamma$ satisfy the reflection law at their common end, and if a linear and a gliding segments of $\gamma$ are successive, then the linear segment is tangent to the gliding one, determining an asymptotic direction for $\partial \Omega_{f}$ at their common end (cf. [7]).

Denote by $\mathscr{V}_{1}$ the set of those $f \in \mathscr{K}$ such that there are no degenerate $(\omega, \theta)$-rays in $\Omega_{f}$ with one vertex. The verification that $\mathscr{V}_{1}$ is open and dense in $\mathbf{C}(X)$ uses some arguments very similar to those below, so we omit the details here.

Let $k>1$, and suppose we have already constructed the sets $\mathscr{V}_{1} \supset \cdots \supset \mathscr{V}_{k-1}$ so that they have the desired properties. Next, we construct $\mathscr{V}_{k}$.

A function $\mathscr{H}:\{1,2, \ldots, k\} \rightarrow\{0,1\}$ is called a $k$-design if $\mathscr{H} \not \equiv 0$, $\mathscr{H}(0)=\mathscr{H}(k)=0$, and $\mathscr{H}(i) \mathscr{H}(i+1)=0$ for each $i=1,2, \ldots, k-2$. If $\gamma$ is a generalized $(\omega, \theta)$-ray with $\operatorname{Im} \gamma=\bigcup_{i=0}^{k} l_{i}$ and $k$ vertices, and for each 
$i=1, \ldots, k-1, \mathscr{H}(i)=0$ holds iff $l_{i}$ is a linear segment, then $\gamma$ is called a ray with design $\mathscr{H}$.

Fix a $k$-design $\mathscr{H}$ and set

$$
\begin{gathered}
q=\max \{i: 1 \leq i \leq k-1, \mathscr{H}(i)=1\}, \\
p=\min \{i: 1 \leq i \leq k-1, \mathscr{H}(i)=1\} .
\end{gathered}
$$

We now use the sets $\mathscr{D}(\omega ; m)$ defined in $\S 2$. Also consider the set $\mathscr{T}_{k}$ of all $f \in \mathbf{C}(X)$ such that there are only finitely many reflecting $(\omega, \theta)$-rays in $\Omega_{f}$ with not more than $k$ vertices and all of them are ordinary. By Theorem 5.1 in [13], $\mathscr{T}_{k}$ contains a residual subset of $\mathbf{C}(X)$. Then by Lemma 2.2 the set:

$$
\mathscr{W}=\mathscr{V}_{k-1} \cap \mathscr{D}(\omega, p) \cap \mathscr{D}(-\theta, q) \cap \mathscr{T}_{k}
$$

also contains a residual subset of $\mathbf{C}(X)$. Fix an arbitrary $r \in \mathbb{N}$ and denote by $\mathscr{V}(k ; r ; \mathscr{H})$ the set of those $f \in \mathscr{W}$ such that there are no generalized $(\omega, \theta)$-rays in $\Omega_{f}$ with design $\mathscr{H}$ and sojourn times $\leq r$.

First, we show that $\mathscr{V}(k ; r ; \mathscr{H})$ in dense in $\mathscr{W}$. To this end we assume id $\in W$, and then we have to prove that there exists $f \in \mathscr{V}(k ; r ; \mathscr{H})$ arbitrarily close to id with respect to the $C^{\infty}$ topology. Observe that there are only finitely many generalized $(\omega, \theta)$-rays in $\Omega_{f}$ with design $\mathscr{H}$ and sojourn times $\leq r$. Indeed, assume there exists an infinite sequence $\left\{\gamma_{m}\right\}$ of distinct generalized $(\omega, \theta)$-rays $\gamma_{m}: \mathbb{R} \rightarrow \Omega$ with design $\mathscr{H}$ and sojourn times $\leq r$. Let $x_{i}^{(m)}=$ $\gamma\left(t_{i}^{(m)}\right), 0=t_{1}^{(m)}<t_{2}^{(m)}<\cdots<t_{k}^{(m)}$, be the successive vertices and $l_{i}^{(m)} \quad(i=$ $0,1, \ldots, k)$ be the successive segments of $\gamma_{m}$. We may assume that there exist $\lim _{m} x_{i}^{(m)}=x_{i}$ for $i=1, \ldots, k$ and $\lim _{m}\left|l_{i}^{(m)}\right| \quad(|l|$ denotes the length of the segment $l$ ) for $i=0,1, \ldots, k$. Then a standard continuity argument shows that for every $t \in \mathbb{R}$ there exists $\lim _{m} \gamma_{m}(t)=\gamma(t)$, and $\gamma$ is a $(\omega, \theta)$-ray in $\Omega$ (cf. [4]). Moreover, $l_{i}=\lim _{m} l_{i}^{(m)}$ are successive segments of $\gamma$ (some of them may consists of only one point so they can be cancelled) with endpoint $x_{1}, \ldots, x_{k}$ and $\operatorname{Im} \gamma=\bigcup_{i=0}^{k} l_{i}$. Since $\theta \neq \omega$ and id $\in \mathscr{W} \subset \mathscr{V}_{1}$, the case $x_{1}=\cdots=x_{k}$ is impossible.

If every $l_{i}$ is nontrivial, i.e. it does not consist of one point, then $\gamma$ would be a generalized $(\omega, \theta)$-ray with design $\mathscr{H}$. Then $\delta_{m}=\bigcup_{i=0}^{p-1} l_{i}^{(m)}$ for any $m \in \mathbb{N}$ and $\delta=\bigcup_{i=0}^{p-1} l_{i}$ are degenerate $\omega$-rays in $\Omega$ with $\delta_{m} \underset{m}{\longrightarrow} \delta$, which is a contradiction with id $\in \mathscr{D}(\omega, p)$. Therefore $l_{i}$ vanishes for at least one $i$. It then follows that $\gamma$ is a reflecting $(\omega, \theta)$-ray in $\Omega$, otherwise we would get a contradiction with id $\in \mathscr{V}_{k-1}$. Clearly, $\gamma$ has at most $k-1$ reflection points. Moreover, applying some arguments similar to those in $\S 4$ of [13], we see that some segment of $\gamma$ is tangent to $X$, which is a contradiction with id $\in \mathscr{T}_{k}$.

Hence there exist only finitely many generalized $(\omega, \theta)$-rays $\gamma, \gamma_{2}, \ldots, \gamma_{n}$ in $\Omega$ with design $\mathscr{H}$ and sojourn times $\leq r$. Let $\operatorname{Im} \gamma=\bigcup_{j=0}^{k} l_{j}$. Then $l_{j}$ is a linear segment iff $\mathscr{H}(j)=0$. Let $x_{i}=\gamma\left(t_{i}\right)$ be the successive vertices of $\gamma$, 
$0=t_{1}<t_{2}<\cdots<t_{k}$. Then

$$
l_{q}=\left\{\gamma(t): t_{q} \leq t \leq t_{q+1}\right\}
$$

is a geodesic on $X$ and $l_{q+1}, \ldots, l_{k-1}$ are linear segments. There is no $a \in$ $\left[t_{q}, t_{q+1}\right)$ such that

$$
\left\{\gamma(t): a \leq t \leq t_{q+1}\right\} \subset l_{s}
$$

for some $s<q$. Indeed, if such $a$ and $s$ exist, then there would be two distinct generalized geodesics in $\Omega$ passing through $x_{q+1}$ in direction $\overrightarrow{x_{q+1} x_{q+2}}$, which is a contradiction with id $\in \mathscr{W} \subset \mathscr{K}$ (cf. [7] or [4]). Hence for every $s=$ $1, \ldots, q-1$ there exists $a \in\left[t_{q}, t_{q+1}\right)$ so that (9) does not hold. Consequently, there is $t_{0} \in\left(t_{q}, t_{q+1}\right)$ such that $\gamma\left(t_{0}\right)$ is not a point of selfintersection of $\gamma$. Moreover, applying the same argument, and eventually replacing $V$ by a smaller neighborhood of $x_{k}$, we see that $t_{0}$ can be chosen so that $\gamma\left(t_{0}\right) \notin$ $\left(\bigcup_{i=2}^{n} \operatorname{Im} \gamma_{i}\right) \cup \bar{V}$. Next, choose a small coordinate neighborhood $U$ of $\gamma\left(t_{0}\right)$ in $X$ with

$$
U \cap\left(\left(\bigcup_{i=2}^{n} \operatorname{Im} \gamma_{i}\right) \cup \bar{V} \cup \bigcup_{\substack{j=0 \\ j \neq q}}^{k} l_{j}\right)=\varnothing
$$

and such that (4) holds for $c(t)=\gamma(t), a=t_{q}, b=t_{q+1}$, and some $\alpha$, $\beta$. By Lemma 3.1 there exists $f \in \mathbf{C}(X)$ arbitrarily close to id such that supp $f \subset U$ and (5) holds for $\widetilde{X}=f(X)$ and the geodesic $\tilde{c}:[a, b] \rightarrow \widetilde{X}$ with $\tilde{c}(t)=c(t)$ for $t \in[a, \alpha]$. Since id $\in \mathscr{D}(\omega ; p)$ it is easily seen that if $f$ is sufficiently close to id, then the only generalized $(\omega, \theta)$-rays in $\Omega_{f}$ with design $\mathscr{H}$ and sojourn times $\leq r$ are $\gamma_{2}, \ldots, \gamma_{n}$ and eventually, a ray $\delta$ with $\delta(0)=x_{1}$ and $\dot{\delta}(0)=\omega$. Assume that for any choice of $f$ there exists such a generalized $(\omega, \theta)$-ray $\delta=\delta_{f}$. Then clearly $\delta(t)=\gamma(t)$ for all $t \leq \alpha$. Let $z_{1}=x_{1}, z_{2}, \ldots, z_{k}$ be the successive vertices of $\delta$. Observe that for $f$ sufficiently close to id the last vertex $z_{k}$ of $\delta$ belongs to $V$. Otherwise we would find a sequence $f_{m} \rightarrow$ id such that the last vertex of $\delta_{m}=\delta_{f_{m}}$ is not contained in $V$ and $\lim _{m} \delta_{m}(t)=\delta(t)$ exists for all $t \in \mathbb{R}$; then $\delta$ would be a generalized $(\omega, \theta)$-ray in $\Omega$ with design $\mathscr{H}$ and sojourn time $\leq r$ different from $\gamma, \gamma_{2}, \ldots, \gamma_{n}$ : a contradiction. Hence $z_{k} \in V$ for $f$ sufficiently close to id, which is a contradiction with the choice of $V$. Thus for $f$ sufficiently close to id there are only $n-1$ generalized $(\omega, \theta)$-rays with design $\mathscr{H}$ and sojourn times $\leq r$ in $\Omega$. Moreover, a simple argument shows that for special construction of $f$ considered above (if $U$ is sufficiently small and $f$ is sufficiently close to id) we have $f \in \mathscr{W}$.

In this way, by induction we construct $g \in \mathscr{V}(k ; r ; \mathscr{H})$ arbitrarily close to id. Hence $\mathscr{V}(k ; r ; \mathscr{H})$ is dense in $\mathscr{W}$. To prove that $\mathscr{V}(k ; r ; \mathscr{H})$ is open in $\mathscr{W}$ it is sufficient to establish that if $\left\{f_{n}\right\} \subset \mathscr{W} \backslash \mathscr{V}(k ; r ; \mathscr{H})$ and $f_{n} \rightarrow$ id $\in \mathscr{W}$, then $X$ admits a generalized $(\omega, \theta)$-ray with design $\mathscr{H}$ and 
sojourn time $\leq r$. This follows easily using some arguments from above and we omit the details.

The set $\bigcap_{\mathscr{H}, r} \mathscr{V}(k ; r ; \mathscr{H})$, where $r \in \mathbb{N}$ and $\mathscr{H}$ runs over the finite set of all $k$-designs, is a residual subset of $\mathscr{W}$; therefore it contains a residual subset $\mathscr{V}_{k}$ of $\mathbf{C}(X)$. Clearly $\mathscr{V}_{k}$ has the desired properties. This completes the construction of the sequence $\left\{\mathscr{V}_{k}\right\}$.

Finally, setting $\mathscr{V}=\bigcap_{k=1}^{\infty} \mathscr{V}_{k}$, we obtain a residual subset of $\mathbf{C}(X)$ such that for any $f \in \mathscr{V}$ there are no generalized $(\omega, \theta)$-rays in $\Omega_{f}$.

Proof of Theorem 1.1. It follows by Theorem 2 in [1] that there exists a residual subset $\mathscr{A}$ of $\mathbf{C}(X)$ such that for any $f \in \mathscr{A}$ and any reflecting $(\omega, \theta)$-ray $\gamma$ in $\Omega_{f}$, if $T_{\gamma} \neq T_{\delta}$ for every generalized $(\omega, \theta)$-ray $\delta$ in $\Omega_{f}$, then $-T_{\gamma}$ belongs to the left-hand side of (2). Set $\mathscr{R}=\mathscr{A} \cap \mathscr{K} \cap \mathscr{V}$, then $\mathscr{R}$ is a residual subset of $\mathbf{C}(X)$. Given $f \in \mathscr{R}$, by [10] or [1] we have that the left-hand side of (2) is contained in the right-hand side. Since $f \in \mathscr{V}$, there are no generalized $(\omega, \theta)$-rays in $\Omega_{f}$, and the above remark implies that (2) holds.

\section{REFERENCES}

1. F. Cardoso, V. Petkov, and L. Stojanov, Singularities of the scattering kernel for generic obstacles, Ann. Inst. Henri Poincaré, Phys. Théorique 53 (1990), 445-466.

2. M. Golubitsky and V. Guillemin, Stable mappings and their singularities, Springer-Verlag, New York, 1973.

3. V. Guillemin, Sojourn time and asymptotic properties of the scattering matrix, Publ. Res. Inst. Math. Sci. 12 (1977), 69-88.

4. L. Hörmander, The analysis of linear partial differential operators, vol. III, Springer-Verlag, Berlin, 1985.

5. E. Landis, Tangential singularities, Funct. Anal. Appl. 14 (1980), 25-34; English Transl. 14 (1980), 98-106.

6. A. Majda, $A$ representation formula for the scattering operator and the inverse problem for arbitrary bodies, Comm. Pure Appl. Math. 30 (1977), 165-194.

7. R. Melrose and J. Sjöstrand, Singularities in boundary value problems. I, Comm. Pure Appl. Math. 31 (1978), 593-617.

8. S. Nakamura, Singularities of the scattering kernel for several convex obstacles, Hokkaido Math. J. 18 (1989), 487-496.

9. S. Nakamura and H. Soga, Singularities of the scattering kernel for two balls, J. Math. Soc. Japan 40 (1988), 205-220.

10. V. Petkov, High frequency asymptotics of the scattering amplitude for non-convex bodies, Comm. Partial Differential Equations 5 (1980), 293-329.

11. V. Petkov and L. Stojanov, Periods of multiple reflecting geodesics and inverse spectral results, Amer. J. Math. 109 (1987), 619-668.

12. __ Spectrum of the Poincare map for periodic reflecting rays in generic domains, Math. Z. 194 (1987), 505-518.

13. __ On the number of periodic reflecting rays in generic domains, Ergodic Theory Dynamical Systems 8 (1988), 81-91. 
14. Singularities of the scattering kernel and scattering invariants for several strictly convex obstacles, Trans. Amer. Math. Soc. 312 (1989), 203-235.

15. T. Wilmore, Introduction to differential geometry, Clarendon Press, Oxford, 1959.

Institute of Mathematics, Bulgarian Academy of Sciences, P. O. Box 373, 1090 Sofia, BUlgaria

Current address: Technische Hochschule Darmstadt, FB Mathematik, Scheossgartenstrasse 7, D-6100 Darmstadt, Germany 